\title{
A JAVA FRAMEWORK FOR ANALYSING AND PROCESSING WOUND IMAGES FOR MEDICAL EDUCATION
}

\author{
Augustin Prodan, Madalina Rusu and Remus Campean \\ Iuliu Hatieganu University \\ Mathematics and Informatics Department \\ Str. Emil Isac 13, Cluj-Napoca 400023, Romania \\ e-mail: \{aprodan, mrusu, rcampean\}@umfcluj.ro
}

\author{
Rodica Prodan \\ MedFam Group \\ Str. Constanta 5, Cluj-Napoca 400158, \\ Romania \\ e-mail: familiaprodan@yahoo.com
}

\section{KEYWORDS}

Active learning, E-learning scenario, Image processing, Java and XML technologies, Wound healing simulation

\begin{abstract}
The paper presents a Java framework for analysing and processing wound images, which are incorporated in elearning tools, to be used by both the students and the teaching staff in their didactic and research activities. We implement in Java traditional methods and algorithms for analysing a digital picture of a wound from a specific distance, for identifying its boundaries and for calculating its area. We build in Java models for various categories of wounds, due to aetiologies such as pressure, burn, chilblain, vascular insufficiencies, diabetic foot ulcer, venous leg ulcer and other chronic disease states. Based on colour and texture analysis, we identify the main barriers to wound healing, such as tissue non-viable, infection, inflammation, moisture imbalance, or edge non-advancing. The Java framework provides the infrastructure for preparing e-learning scenarios based on practice and real world experiences. Also, we rely on new paradigms of artificial intelligence (Bayesian Inference, Case Based Reasoning and Intelligent Agents) for creating e-learning scenarios to be used in a context of active learning. To implement these e-learning tools, we use Java technologies for dynamic processes and XML technologies for dynamic content (data and documents).
\end{abstract}

\section{INTRODUCTION}

Medical images are valuable in both didactic and research activities, for students in medicine and pharmacy. Digital pictures are in great demand, because digital technologies provide unlimited resources for medical and pharmaceutical education. Computerized image processing contains methods for non-invasive wound evaluation, allowing an accurate diagnosis in a large category of patients with damaged and ulcered skin. Traditional non-invasive technologies are limited frequently to subjective visual evaluations. Colour and texture information provide the infrastructure for a structured approach to non-invasive wound assessment. As presented in (Hansen et al. 1997), statistical methods are useful in assessment of potential methodologies for non-invasive wound evaluation using a colour imaging system. In (Krouskop et al. 2002) is described a non- contact wound measurement system for surface area and volume of wounds. A classification method based on colour and texture analysis is proposed in (Acha and Serrano 2000), being applied for burn wound images.

This paper presents a Java framework for analysing, processing and understanding wound images. This represents our initial work towards a model of colour and texture based simulation for wound healing. We use the RGB (Red-Green-Blue) colour space to define a set of image features for every category of wounds. To identify a wound in an image, we implemented specific methods based on some generic criteria, such as normal skin, wound skin, yellow wound, black wound, red wound, etc. For some applications we use as main colours Red, Yellow and Black to asses the gravity of a wound. Generally, wounds have a non-uniform mixture of yellow slough, red granulation tissue and black necrotic tissue. Analysing a succession in time of more images for the same wound, we can assess the recovery or worse evolution.

Traditionally, programmers use $\mathrm{C}, \mathrm{C}++$ and $\mathrm{C} \#$ to develop applications for image processing. However, if we have to analyse and process images in a Web context, the best solution is to manage it in Java. Our purpose is to develop e-learning tools for students and residents in medicine and pharmacy, to be used in both didactic and research activities. In (Prodan et al. 2003) we defined and implemented a Java framework for designing and implementing e-learning scenarios. We used this framework to develop e-learning tools to be used by students and residents to learn biostatistics, in a context of open learning. We have to extend this system with specific e-learning scenarios for medical education. Our aim is to create and implement in Java an automatic method which can be used as a reference standard for colour and texture wound analysis. We have to create elearning scenarios by applying this method to large amounts of wound image data stored in XML based knowledge data (Figure 1). 




Figure 1: General method for creating the e-learning scenarios

The objective is to develop appropriate skills in wound management for a learner that traverses such an elearning scenario. The e-learning scenarios are practice driven and relevant to professional practice, being used by students in medicine and pharmacy, at graduate, postgraduate and residency levels. Wound image understanding is a difficult knowledge-based process and we have to use the new paradigms of Artificial Intelligence (e.g. Bayesian Inference, Case Based Reasoning and Intelligent Agents) to manage it. We collected large amounts of wound image data and we have to include them in XML and CBR (Case Based Reasoning) knowledge bases. We are working in a continuous collaboration with physicians and wound care experts from our university and from health care and medical units. We have continuous access to actual medical records to have in view the wound evolution and to verify both the accuracy and the consistency of our system. The advantage of using Java for this purpose is the integration without any difficulty with other Web based facilities.

\section{METHODS FOR IMAGE PROCESSING}

We are creating a collection of Java class libraries, implementing methods for processing images corresponding to various categories of wounds, due to aetiologies such as pressure, burn, chilblain, vascular insufficiencies, diabetic foot ulcer, venous leg ulcer and other chronic disease states. We implement general methods that create many common special effects, such as the following:

- Control the brightness and the contrast of an image;

- Highlight a particular area in an image;

- Sharpen all or part of an image;

- Blur all or part of an image;

- Apply colour filtering to an image;

- Perform edge detection on an image;

- Morph one image into another image;

- Apply colour inversion to an image;

- Change the size of an image;

- Create a kaleidoscope of an image;

- Squeeze part of an image into a smaller one;

- Deal with the effects of noise in an image.

A digital image consists of a two dimensional array of pixels $P_{m n}$ with $m$ rows and $n$ columns. We represent an image in internal memory as a three dimensional array
$P_{m n 4}$, each pixel being described in a specific RGB format (Prodan and Prodan 1997) by four unsigned 8-bit integers. The first three integers represent the base colour components (Red, Green and Blue), and the fourth integer, referred to as $\alpha$ (alpha) represents the transparency. A specific colour is obtained by mixing different amounts of basic colours (red, green and blue) with a specific transparency. The standard Core Java Technologies provides methods for processing digital images. The package java.awt.image contains methods that allow to blur, sharpen, brighten or tone down an image. The class ConvolveOp implements a convolution from a source to a destination image, replacing each pixel with some combination of the original pixel and its neighbours. We will create the Java framework by implementing the image processing algorithms into one of the following two layers:

- Layer 1 - which contains the low-level implementations, allowing to operate directly on pixels.

- Layer 2 - which contains the high-level implementations, based on standard Java libraries such as JAI (Java Advanced Imaging) API.

For each wound we have to find out some quantitative and qualitative attributes for assessing the healing state. As quantitative attributes we have to measure its surface area and its volume (evaluating depth). We process the original image with the purpose to emphasize the distinction between wound and non-wound area. We use some general methods to enhance the image, because we must exaggerate the distinction between wound and non-wound. As an example, for individuals with fair skin, we lighten the images and then view them using shades of green with the red and blue minimized. This way more clearly exhibit the borders of the wound than in the original image. Removal of the red and blue leaves the wound black and the rest of the image green. For images of individuals with dark skin, both the red and green are accentuated while the blue is minimized. This procedure also leaves the non-wound area green, but colours the wound red. In either case, the wound can easily be distinguished from the non-wound without difficulty. We have to implement e-tools that will enable to assess the current state of the wound and to gain insight into the wound evolution, by comparing the series of wound data collected over time. Based on this knowledge we can design an e-tool for simulating the process of wound healing. Based on this knowledge we can design an e-tool for simulating the process of wound healing.

\section{CLASSIFICATION METHODS IN JAVA}

We are working in a continuous collaboration with physicians and wound care experts to make a rigorous classification for various categories of wounds. We collected large amounts of wound image data and we calculate statistical parameters as mean, median, standard deviation, confidence interval, skewness and kurtosis for them. We include these historical data in 
XML based databases, to be used as inputs to classification algorithms. Our purpose is to make distinction between infected and non-infected, inflamed and non-inflamed wounds. Based on colour analysis, we build a statistically significant differentiation of mild, moderate and severe wounds. We analyse the differences in calibrated hue between injured and noninjured skin, obtaining a repeatable differentiation of wound severity for various time intervals. As an example, burn wounds are characterized according to their depth as:

- Superficial - with bright red colour and the presence of blisters (usually brown colour);

o Deep - with red-whitish colour and with dark dots;

○ Full thickness - with creamy with or dark brown colour.

We implement classification methods to classify wound images into different groups based on colour and texture information. We investigated the suitability of statistical parameters for providing useful inputs to the classification algorithms.

\section{Mean and standard deviation}

Assuming normality, the first two moments (mean and standard deviation) characterize very well the colour distribution. The mean represents the centre point of the distribution, separating the values into two equally probable subsets. Standard deviation represents the dynamics of the values, how wide around the mean the colours of the wound image are distributed. We use the first two moments (mean and standard deviation) to modify the contrast and the brightness of an image. The contrast is determined by the width of the distribution, while the brightness is determined by the location of the grouping colour values (Baldwin, 2005). We implemented Java programs that use the mean and standard deviation to modify and control both the contrast and the brightness of an image, by modifying the distribution of the colour values.
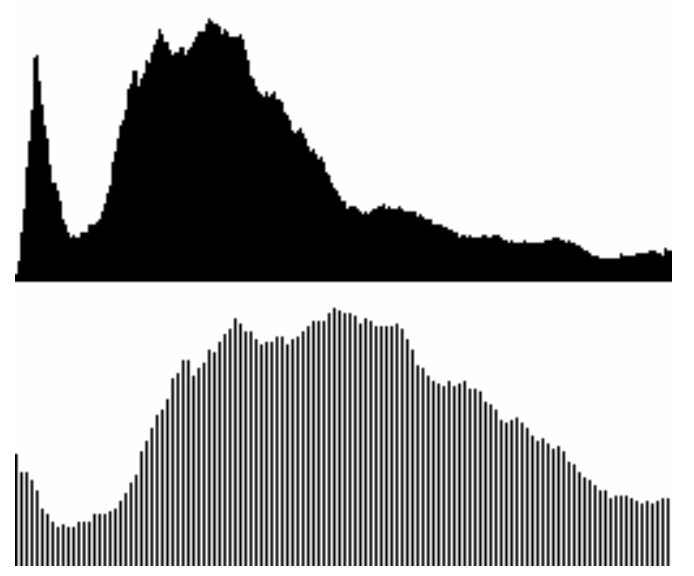

Figure 2: The distribution of the colour values before processing (up) and after processing (down).
Figure 2 shows the distribution of the colour values contained in the original image (up), compared with the distribution contained in the modified image (down). In processed image, the contrast (width of the distribution) is increased by a factor of 2.0 and the brightness (mean value) was increased by a factor of 1.7 .

\section{Skewness and kurtosis}

Sometimes the first two moments alone are inadequate to discriminate between wound and non-wound skin. Therefore further details of the colour distribution are required. Skewness and kurtosis of the colour data proved to be more useful for this purpose.

Skewness represents the symmetry of the distribution around the centre. Skewness is null for a normal distribution, positive when the distribution is skewed right (i.e. when the upper tail is predominant) and negative when the distribution is skewed left.

If $x$ is a random variable, we define the skewness $\gamma_{3}$ as the normalized third order moment, in the following way:

$\gamma_{3}=\frac{E\left[(x-E[x])^{3}\right.}{\left(E\left[(x-E[x])^{2}\right]\right)^{3 / 2}}$

For a sample $x_{i}$, an estimate of the skewness is given by:

$$
g_{3}=\frac{\sum_{i=1}^{N}\left(x_{i}-m\right)^{3}}{(N-1) s^{3}}
$$

where $m$ and $s$ are estimates of the mean and the standard deviation.

Kurtosis quantifies the flatness level of the distribution at the mean. Kurtosis is equal to 3 for a normal distribution. If kurtosis is lower than 3 , the distribution is said to be platokurtic (i.e. wide-peaked) and if kurtosis is higher than 3 , thae distribution is said to be leptokurtic (i.e. narrow-peaked). The value 3 may be subtracted as an offset, as in the following formulae.

For the same random variable $x$, kurtosis $\gamma_{4}$ is the normalized fourth order moment, being defined as:

$\gamma_{4}=\frac{E\left[(x-E[x])^{4}\right]}{\left(E\left[(x-E[x])^{2}\right]\right)^{2}}-3$

The kurtosis is used as a measure of the heaviness of the tails in a distribution. For a sample $x_{i}$, an estimate of the kurtosis is given by: 
$g_{4}=\frac{\sum_{i=1}^{N}\left(x_{i}-m\right)^{4}}{(N-1) s^{4}}-3$

We have to build in Java models for various categories of wounds, due to aetiologies such as pressure, burn, chilblain, vascular insufficiencies, diabetic foot ulcer, venous leg ulcer and other chronic disease states. Based on colour and texture analysis, we have to identify the main barriers to wound healing, such as tissue nonviable, infection, inflammation, moisture imbalance, or edge non-advancing. Our aim is to implement algorithms for wound healing simulations.

\section{IDENTIFYING THE WOUND}

The first task we face with in our system is to identify the wound in a digital image. For this purpose, we implemented specific methods based on some generic criteria, such as normal skin, wound skin, yellow wound, black wound, red wound, etc. We incorporated these methods in applications endowed with friendly GUI (Graphical User Interface). When an application is launched, it makes the following general actions:

1. Reads the digital image in main memory;

2. Convert pixel data of the digital image into a threedimensional array that is better suited for processing;

3. Make a working copy of the three-dimensional array, in order to avoid having to make changes to the original array of pixel data. The working copy is sacrificed in the process of analysing the image, while the original image rest unchanged;

4. Display on the same frame both the original image and the modified image that contains the output results.

It is not difficult for a user to identify interactively a wound making the following actions:

1. Select a representative area for the wound;

2. Select a representative area for the normal skin;

3. Push a button to begin the analyse process.

As a general approach, we divide the image into little boxes and then we start analyzing each box for colour profile (percentage of main colours). We examine the difference in the colour profile of the examined box to the colour profile of a box covering healthy skin, taken from outside the wound area. The distribution obtained from a box with healthy skin can be used as a benchmark. Other distributions are then compared in statistical terms with this baseline distribution and decisions are made on determining the edge. Wound area and different colour percentages follow from this as well. The degree of deviations from this benchmark distribution can then be used to classify wounds. Assuming normality, the first two moments (the mean and the standard deviation) estimated from a sample will determine the colour/texture distribution. The edge identification has an element of subjectivity which is left to the medic or wound specialist to set. Say for example, that wound edge starts if the colour profile changes $40 \%, 70 \%$ or $90 \%$, depending on how sensitive we want the detector to be.

As an example, Figure 3 shows the output result displayed by such an application launched to identify a wound. The left side contains the original image, while the right side contains the processed clone image showing wound area marked with a specific colour.

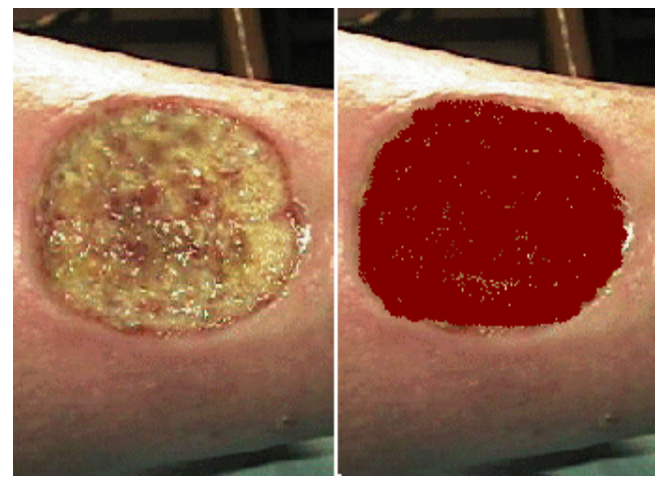

Figure 3: The output result of identifying a wound

We apply two strategies his work of identifying the wounds: a global strategy and a wound by wound strategy. The user may choose one of the two strategies, or may combine them using the GUI facilities.

\section{Global strategy}

When apply this strategy, the whole image is traversed from top-left corner towards bottom-right corner, applying specific methods for edge-detection and wound identification. The output result is the identification of all wounds in current image.

\section{Wound by wound strategy}

When apply this strategy, each wound is identified in a separate process, based on a representative area belonging to it. In this case, only the selected wound is traversed, starting with representative area and going towards the four main points: top-left, top-right, bottomleft and bottom-right.

\section{E-LEARNING ENVIRONMENT}

We defined and implemented a Java framework for designing and implementing intelligent and practical elearning tools, to be used by both the students and the teaching staff in a context of open learning (Prodan and Prodan, 2003). This framework provides the infrastructure for preparing e-learning scenarios based on practice and real world experiences, as practice is essential in learning activities. Our e-learning scenarios promote active learning, forcing the students to take part in real world activities simulated on computer. Also, we designed e-learning tools based on bootstrapping methods (which are quite valuable for 
reasoning in uncertain conditions), with the purpose to simulate laboratory experiments in both didactic and research activities (Prodan and Campean, 2004, 2005). The Java framework provides the infrastructure for preparing e-learning scenarios based on practice and real world experiences. Also, we rely on new paradigms of artificial intelligence (Bayesian Inference, Case Based Reasoning and Intelligent Agents) for creating elearning scenarios to be used in a context of active learning. An e-learning scenario combines simulation and interactive visualization and allows the learners to explore the knowledge bases with some well-defined learning purposes. We define a simulation class and a visualization class for each application object. These classes are then configured to obtain a particular simulation with a specific visualization. In an e-learning scenario, visualization is an active part of the system, serving as an additional interface for modifying dynamically some parameters. We write all simulation and visualization classes in Java and use the XML format to describe the configurations, defining both the components and their relationships. An e-learning scenario is in fact like a traditional lesson, and the ideal solution is to simulate a teaching-learning relation with a virtual teacher able to interact with the learners and to instruct them. A good traditional teacher learns all the time from previous didactic experiences. Based on this historical feedback, the teacher exploits prior specific successful episodes, and avoids prior failures. We introduce a similar feedback mechanism in our technology of elaborating e-courses (Figure 4). Following the traditional model, we store cases of positive experiences from previous e-learning scenarios in case bases created with XML and CBR technologies (Leake, 1996).

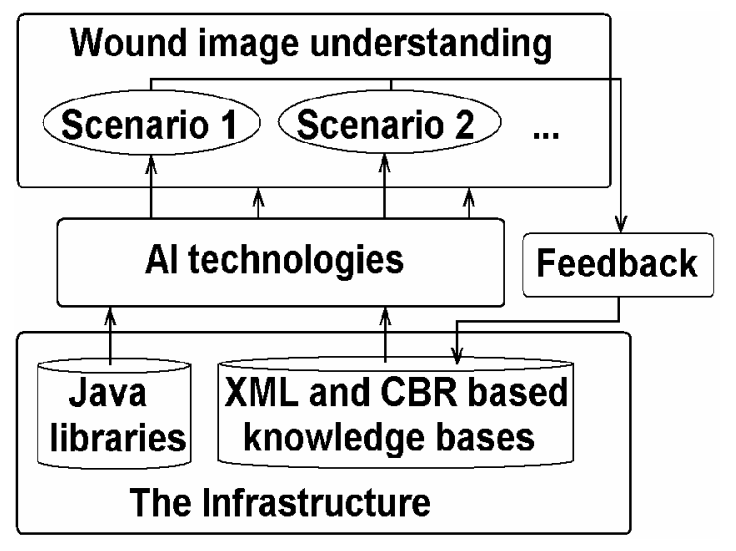

Figure 4: The Generation of the E-learning Scenarios

We have to extend this e-learning system with specific e-learning scenarios for medical education. Our aim is to create and implement in Java an automatic method which can be used as a reference standard for colour and texture wound analysis. We have to create e-learning scenarios by applying this method to large amounts of wound image data stored in XML based knowledge data. By estimating the percentages for the main colours of red, yellow and black, is possible to assess the gravity of the wound. The image processing program allows the user to interactively control the process. The user can set the tolerance for each colour, that is the width of the band of acceptable colours. Based on colour analysis and statistical methods, we can analyse successive states of a wound, assessing the healing or worsen evolution. We develop a flexible and adaptable system for wound image understanding, based on new paradigms of Artificial Intelligence (e.g. Bayesian Inference, Case Based Reasoning and Intelligent Agents). The functionality of this system will be used for creating elearning tools, to be used by the students in medicine and pharmacy, at graduate, postgraduate and residency levels, for developing appropriate skills in wound management (Figure 4). We are working in a continuous collaboration with physicians and wound care experts from our university and from health care and medical units. We have continuous access to actual medical records to have in view the wound evolution and to verify the accuracy and the consistency of our system. We have to compare all the time the observed and the estimated values of the colour with each other. Based on colour and texture analysis, we have to identify the main barriers to wound healing, such as tissue non-viable, infection, inflammation, moisture imbalance, or edge non-advancing. Our aim is to implement algorithms for wound healing simulations. The advantage of using Java for this purpose is the integration without any difficulty with other Web based facilities.

\section{CONCLUSIONS}

This paper presents a Java framework for analysing and processing wound images, to be used in teaching, learning and research activities. The colour image processing methods have many advantages over traditional human methods in assessment of wounds. Computer based methods are objective, repeatable and with a large potential of processing. The analysis of a wound from a specific distance involves procedures devoted to identify its boundaries, to calculate its area and to estimate proportions of the main colours red, yellow and black. Generally, wounds have a nonuniform mixture of yellow slough, red granulation tissue and black necrotic tissue. To analyse the actual state of the wound and the healing evolution, it is necessary to determine the proportions of these main colours. We have to create XML based databases containing knowledge extracted from previous wound healing experiences and from medical experts knowledge. As a future work, we have to implement e-learning tools and e-learning scenarios enabling to perform quantitative measurements of wound evolution in time and to asses the healing or worsen. This is our initial work towards a model of colour and texture based simulation for the wound healing. We have to simulate wound healing based on various treatments and to compare the results with experimental observations 


\section{REFERENCES}

Acha, B. and Serrano, C. 2000. "Image Classification Based on Color and Texture Analysis". First Int'l Workshop on Image and Signal Processing and Analysis, June 14-15, 2000, Pula, Croatia, 95-99.

Baldwin, R. G. 2005. "Processing Image Pixels using Java: Controlling Contrast and Brightness". Available at address: http://www.developer.com/java/other/article.php/3441391.

Hansen, G.L.; Sparrow, E. M.; Leland, K. J. and Iaizzo, P. A. 1997. "Wound status evaluation using image processing". IEEE Transactions on Medical Imaging, Vol. 16, No. 1 (February), 78-86.

Krouskop, T. A.; Baker, R. and Wilson, M.S. 2002. "A noncontact wound measurement system". Journal of Rehabilitation Research and Development, Vol. 39, No. 3 (May/June), 337-346.

Leake, D. P. (1996). Case-Based Reasoning: Experiences, Lessons, and Future Directions (Menlo Park: AAAI Press/MIT Press).

Prodan, A. and Prodan, M. 1997. Java Environment for Internet, Editura Promedia-Plus, Cluj-Napoca, 1997, ISBN 973-9275-07-09.

Prodan, A., Prodan, R. 2003. "A Java framework for intelligent and practical e-learning tools", In Fernstrom, K. (Ed.) Proceedings of the $4^{\text {th }}$ International Conference on Information Communication Technologies in Education), ISBN 960-8313-16-3, ISSN 1109-2084, Samos Island, 3-5 July, (45-52).

Prodan, A., Campean, R. 2004. "Bootstrapping e-tools for simulating laboratory works", In Fernstrom, K. (Ed.), Proceedings of the $5^{\text {th }}$ International Conference on Information Communication Technologies in Education, (pp. 489-494), ISBN: 960-8313-85-6, ISSN:1109-2084, Samos Island, 1-3 July, University of Athens.

Prodan, A., Campean, R. 2005. "Bootstrapping methods applied for simulating laboratory works", Campus-Wide Information Systems, 22 (3), 168-175, Emerald, ISSN 1065-0741, ISBN 1-84544-399-X.

\section{AUTHOR BIOGRAPHIES}

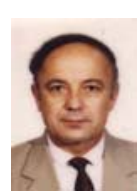

AUGUSTIN PRODAN, born in VarJibou, Salaj, Romania and went to the Babes-Bolyai University of Cluj-Napoca, where he studied Mathematics and Informatics and obtained his degree in 1968. $\mathrm{He}$ worked for 27 years at Computer Science Research Institute (Software ITC), Department of Cluj-Napoca. He managed the software teams to implement traditional programming languages (COBOL, FORTRAN, PASCAL, C) and he made courses and software assistance for romanian and foreign users (China, Czechoslovakia, East Germany, Hungary and Russia). In 1988 he obtained the $\mathrm{PhD}$ in Mathematics and Informatics at the
Babes-Bolyai University. Since 1995 he is professor at Iuliu Hațieganu University, head of Mathematics and Informatics Department. He makes courses of Biomathematics, Biostatistics and Informatics, at graduate, resident and postgraduate levels, for Romanian and French sections. The research domains: Stochastic Modelling and Simulation, Java Based Technologies, e-Learning, Artificial Intelligence, Intelligent Agents, Bayesian Inference, Biomathematics and Bioinformatics, Data Mining, Web-based Databases, Semantic Web, XML, Knowledge Engineering and Management, Image Processing. e-mail: aprodan@umfcluj.ro; Web page: http://freewebs.com/augustinprodan/

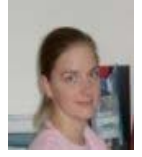

MADALINA RUSU, Master of Science in Informatics and Medical Informatics, $\mathrm{PhD}$ student in Medical Databases at BabesBolyai University, Cluj-Napoca, Romania, Faculty of Mathematics and Informatics. Research directions: Medical databases, Web applications, Medical education.

e-mail: mrusu@umfcluj.ro ;

Web page: www.flowerpower.i8.com.

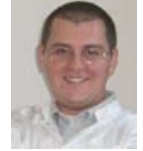

REMUS CAMPEAN, Master of Science in Biostatistics and Medical Informatics, $\mathrm{PhD}$ student in Applied Mathematics, Numerical and Statistical Methods, at Babes-Bolyai University, Cluj-Napoca, Romania, Faculty of Mathematics and Informatics. Research directions: Modelling, simulation, Optimization and control in pharmaceutical and biomedical sciences.

e-mail: rcampean@umfcluj.ro;

Web page:

http://www.freewebs.com/remus campean/

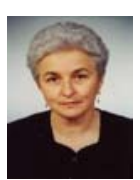

RODICA PRODAN, born in Jibou, Salaj, Romania and went to Iuliu Hatieganu University of Cluj-Napoca, where she studied Medicine and obtained her degree in 1971. She worked some years in Jibou and Turda as general practitioner in the railway medical system. Based on a professional competition, Rodica Prodan entered in Cluj-Napoca as general practitioner. With her rich experience as general practitioner, Rodica Prodan contributed to researches in various medical domains and is co-author for many scientific articles. e-mail: familiaprodan@yahoo.com. 